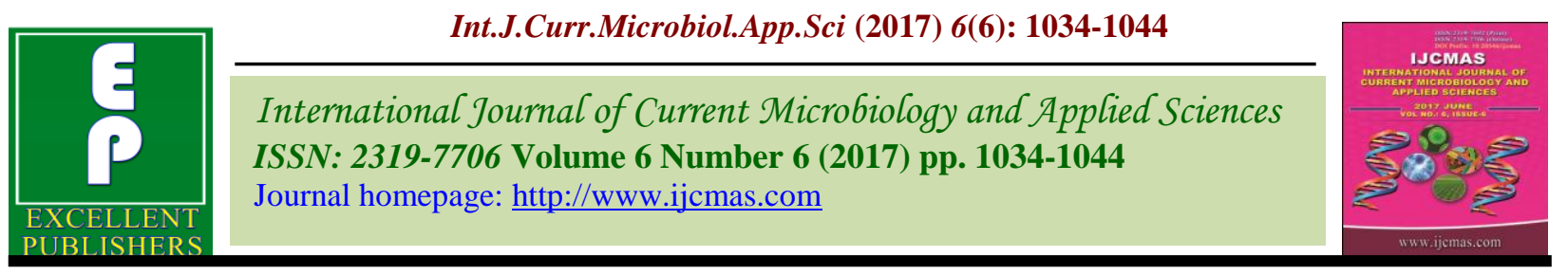

Original Research Article

https://doi.org/10.20546/ijcmas.2017.606.119

\title{
Molecular Marker based Genetic Diversity Analysis in Soybean [Glycine max (L.) Merrill] Genotypes
}

\author{
Ravindra Kumar Jain*, Arunabh Joshi and Devendra Jain
}

Department of Molecular Biology and Biotechnology, Rajasthan College of Agriculture, Maharana Pratap University of Agriculture and Technology, Udaipur 313001, (Rajasthan), India

*Corresponding author

\section{A B S T R A C T}

Soybean is an important legume and oilseed crop with high protein (40\%) and oil

Keywords

Soybean, RAPD,

Genetic diversity, Polymorphism, PIC,

Genetic variability,

Similarity coefficient.

\section{Article Info}

Accepted:

17 May 2017

Available Online:

10 June 2017
(20\%). RAPD markers were used to access the genetic diversity among twenty four soybean genotypes. A total of Twenty primers were used out of which 18 got amplified which produced 164 bands and all were found polymorphic i.e. 100\% polymorphism. The total number of amplified bands varied between 2 (OPF-19) and 16 (OPA-01) with an average of 9.1 bands per primer. The overall size of the amplified fragments ranged between 100 and $2500 \mathrm{bp}$. The Polymorphic Information Content (PIC) values ranged from 0.126 (OPP-01) to 0.399 (OPF-19) with an average of 0.295 . Jaccard's similarity coefficient values ranged from 0.12 to 0.70 with an average of 0.41 . Cluster analysis based on Jaccard's similarity coefficient using Un-weighted Pair Group Method with Arithmetic Averages (UPGMA) grouped all the 24genotypes into three major groups at a similarity coefficient of 0.53 . A total of four primers detected in the study produced four unique bands in four genotypes. The results showed that the level of genetic variation was high among the soybean genotypes.

\section{Introduction}

Soybean (Glycine $\max$ (L.) Merrill) is a diploidized, allotetraploid $\quad(2 \mathrm{n}=40)$, autogamous plant belongs to legume family. It has originated in the eastern half of North China in the 11th century B.C. or perhaps a bit earlier (Fukuda, 1933 and Singh, 2010). This crop is aptly called as "Golden Bean" or "Miracle crop" of the 20th century, because of its multiple uses. It is a principle grain legume in developing countries where it meets the expanding needs for protein, edible oil and calories. It contains $40-42 \%$ protein, $18-22 \%$ oil comprising of $85 \%$ unsaturated fatty acids and $15 \%$ saturated fatty acids, $28 \%$ carbohydrate and good amount of other nutrients like phosphorus, calcium, vitamins, iron etc. (Antalina, 1999) and rich in lycine and vitamin A, B and D. It also consist many therapeutic components and has increased its importance in industrial, agricultural and medicinal sectors.

Genetic diversity evaluation among germplasms is an important and a prerequisite in any hybridization program and would promote the efficient use of genetic variations 
(Paterson et al., 1991; Chen et al., 1994; Dwivedi et al., 2001). The assessment of genetic diversity is important not only for crop improvement but also for efficient management and conservation of germplasm resources (Tahir and Karim, 2011). Marker systems have been successfully used over the last several decades to construct genetic maps, assess genetic diversity and locate genes of interest in a number of agriculturally important crops for the desired traits (Garcia et al., 2005). Different methodological approaches such as morphological, protein, Isozyme and molecular markers have been employed to assess genetic diversity in crop plants. Among them, the DNA based molecular marker approach has been found to be superior, because of its capability to reveal more polymorphism (Mignouna et al., 1998). Molecular markers have been proved to be valuable tools in the characterization and evaluation of genetic diversity within and between species and population.

RAPD markers offer many advantages such as higher frequency of polymorphism, rapidity, technical simplicity, requirement of a few nanograms of DNA, no requirement of prior information of any DNA sequence and feasibility of automation (Fahima et al., 1999; Subudhi and Huang, 1999; Chowdhury et al., 2001; Zenglu and Nelson, 2002; Yu et al., 2005; Kumari et al., 2009). The RAPD technique which was developed by Williams et al., (1990) has been widely applied in either identification of cultivars $(\mathrm{Hu}$ and Quiros, 1991) or estimating genetic relationship and diversity among crop germplasm (Jain et al., 1994).

RAPD markers have been used for genetic diversity analysis in soybean by many workers (Thompson and Nelson, 1998; Thompson et al., 1998, Brown-Guidera et al., 2000; Li et al., 2001; Li and Nelson, 2001; Singh et al., 2006; Ojo et al., 2012; Khare et al., 2013; Bharose et al., 2017). In the present work, we have applied RAPD markers to characterize and assess the genetic variability in selected 24 soybean genotypes and to determine the phylogenetic relationship among them.

\section{Materials and Methods}

Twenty four genotypes of soybean were procured and investigated in the present study (Table 1). Young fresh and healthy leaves were collected and DNA extraction was done following the cetyl trimethyl ammonium bromide (CTAB) method (Doyle and Doyle, 1990). The extracted DNA was analysed on $0.8 \%$ agarose gel and was diluted to an optimum concentration using $\mathrm{TE}$ for polymerase chain reaction (PCR). A total of 20 arbitrary decamer primers were initially used, out of which 18 primers showed clear, scorable and highly polymorphic bands (Table 2).

Different parameters were tested to determine optimal concentrations of template DNA, $\mathrm{MgCl}_{2}$, dNTPs, Taq DNA polymerase, primer and different temperatures and time intervals during denaturation, annealing and elongation steps which affect amplification, banding pattern and reproducibility. For this, varying concentrations of template DNA (50 ng, 100 ng, $200 \mathrm{ng})$, primers $(0.10 \mu \mathrm{M}, 0.20 \mu \mathrm{M}, 0.30$ $\mu \mathrm{M}, 0.40 \mu \mathrm{M}, 0.50 \mu \mathrm{M})$, dNTPs $(0.5 \mathrm{mM}, 1$ $\mathrm{mM}, 1.5 \mathrm{mM}, 2.0 \mathrm{mM})$ and $\mathrm{MgCl}_{2}(0.5 \mathrm{mM}$, $1.0 \mathrm{mM}, 1.5 \mathrm{mM}$ and $2.0 \mathrm{mM}$ ) were used in a reaction volume of $20 \mu \mathrm{l}$ in different combinations at different annealing temperatures $\left(38^{\circ} \mathrm{C}, 40^{\circ} \mathrm{C}, 43^{\circ} \mathrm{C}, 45^{\circ} \mathrm{C}\right.$, and $\left.48^{\circ} \mathrm{C}\right)$. In brief, reproducible and clear banding patterns were obtained in a reaction mixture of $20 \mathrm{ml}$ containing $1 \mathrm{x}$ reaction buffer, 1 unit of Taq DNA polymerase, 200 $\mathrm{mM}$ each of dNTPs mix, $0.5 \mu \mathrm{M} /$ reaction of primer's and $50 \mathrm{ng}$ of template DNA. 
The Polymerase Chain Reaction was performed in a programmable thermo-cycler DNA Engine (Biorad, Germany) using the following cycling parameters: an initial denaturation $\left(94^{\circ} \mathrm{C}\right)$ for 5 minutes, Denaturation $\left(94^{\circ} \mathrm{C}\right)$ for 2 minutes, Primer annealing $\left(36^{\circ} \mathrm{C}\right)$ for 1 minute, Primer Extension $\left(72^{\circ} \mathrm{C}\right)$ for 2 minutes (37 cycles), followed by Final Primer Extension $\left(72^{\circ} \mathrm{C}\right)$ for 10 minutes and a hold temperature of $4^{\circ} \mathrm{C}$.

The amplified products, after PCR reaction, were separated on $1.2 \%$ agarose gel in $1 x$ TAE buffer using ethidium bromide(EtBr) staining dye. The size of the amplified DNA fragments was determined using $100 \mathrm{bp}$ and 1 kb DNA ladders (Bangalore Genie, India) as standard markers. DNA fragments were visualized under UV-trans-illuminator and photographed using gel documentation system. Scoring of amplicons obtained from different RAPD markers was done on the basis of presence (used as 1) or absence (used as 0) of bands for each primer. For banding pattern only clear and unambiguous bands were scored for each primer. Comparison of band position was done with molecular weight of standard DNA ladders. Accordingly, a rectangular binary matrix was obtained and statistical analysis was performed using the NTSYS-pc version $2.02 \mathrm{e}$ (Rohlf, 1998). A pair wise similarity matrix was generated and the cluster analysis was performed via Unweighted Pair Group Method with Arithmetic averages (UPGMA) to develop a dendrogram. A two dimensional and three dimensional principal component analysis (PCA) was constructed to provide another means of testing the relationship among the genotypes.

\section{Results and Discussion}

Among the 20 RAPD primers used for initial screening, 18 markers produced polymorphic, reproducible and scorable bands. A total of
164 amplified bands were obtained of which all were polymorphic and showed $100 \%$ polymorphism (Table 2). The total number of amplified bands varied between 2 (primer OPF-19) and 16 (primer OPA-01) with an average of 9.1 bands per primer. The overall size of PCR amplified products ranged between $100 \mathrm{bp}$ to $2500 \mathrm{bp}$. The percent polymorphism was $100 \%$ for all the genotypes. The average Polymorphic Information Content (PIC) was 0.295 ranging from 0.126 (OPP-01) to 0.399 (OPF-19). Figure 1 showing the amplification pattern obtained from primer OPP-01 and OPP-04 produced 10 and 14 polymorphic band respectively.

Four unique bands (band which is present in a particular genotype but absent in rest of the genotypes) were detected in four genotypes viz., JS-20-79, PS-1543, Himso-1685 and NRC-98 with 4 RAPD primers (OPJ-04, OPP-05, OPP-06 and OPD-05). All four genotypes gave single distinct bands. The size of these unique bands ranged from 200-2100 bp (Table 3).

The data obtained by using RAPD were further used to construct similarity matrix using 'Simqual' sub-programme of software NTSYS-pc. Based on RAPD similarity matrix data, the values of similarity coefficient ranged from 0.12 to 0.70 i.e. $12-70 \%$ or genetic diversity ranged from 30 to $88 \%$ (Table 4). The average similarity across all the genotypes was found out to be 0.41 showing that the genotypes were highly diverse from each other. The maximum similarity coefficient 0.70 was observed between SL-983 and DS-2961 and RVS 2002-22 and RKS-111 showing minimum diversity followed by PS-1543 and Himso1685 and KDS-722 and MAUS-609 with a similarity coefficient value of 0.69 and 0.68 respectively. The minimum similarity coefficient 0.12 was observed between PS- 
1539 and MACS-1419 indicating maximum diversity followed by PS-1539 and NRC-98, PS-1539 and MACS-1410 and MACS-1419 and BAUS-27 with a similarity coefficient of 0.14 .

The RAPD cluster tree analysis of 24 G. max L. genotypes showed that they could be mainly divided into 3 major clusters at a similarity coefficient of 0.29 (Fig. 2). Cluster I included 12 genotypes viz., KDS-726, DS3050, SL-983, DS-2961, AMS-1001, JS-2079, RKS-109, DS-3047, RVS-2002-4, KDS722, MAUS-609 and PS-1539 at a similarity coefficient of 0.31 . It could be divided into 2 sub-clusters. The sub-cluster first contained two genotypes SL-983 and DS-2961 similar to each other at a very close to 0.70 similarity coefficient while the second sub-cluster consisted two genotypes KDS-722 and MAUS-609 that are related to each other at 0.68 similarity coefficient. Cluster II included 8 genotypes at a similarity coefficient of 0.42 . These genotypes are MACS-1410, PS-1543, Himso-1685, RVS-2002-22, RKS-111, JS-2053, RSC-10-17 and BAUS-27. This cluster could be further divided into two sub-clusters.

Table.1 Pedigree and source of 24 genotypes of Glycine max L. Merrill

\begin{tabular}{|c|c|c|c|}
\hline S.No. & Genotypes & Pedigree & Source \\
\hline 1. & KDS-726 & JS-93-05 X EC-241780 & SANGLI (MH) \\
\hline 2. & PS-1539 & PS-1024 X JS-335 & PANTNAGAR \\
\hline 3. & DS-3050 & DT-23 X DT-227 & DELHI \\
\hline 4. & SL-983 & SL-525 X PK-1368 & LUDHIANA \\
\hline 5. & DS-2961 & MO-74 X JS-335 & DELHI \\
\hline 6. & RKS-109 & RKS-224 X PK-1024 & KOTA \\
\hline 7. & SL-955 & SL-599 X PK-1283 & LUDHIANA \\
\hline 8. & DS-3047 & DT-23 X DT-27 & DELHI \\
\hline 9. & AMS-1001 & Mutant of JS-93-05 & AMARAWATI \\
\hline 10. & JS-20-79 & JS-97-52 X JS-(15) 90-5-12-1 & JABALPUR \\
\hline 11. & MACS-1419 & EC-391343 X MACS-450 & PUNE \\
\hline 12. & NRC-98 & Ankur X PK-1024 & INDORE \\
\hline 13. & RVS-2002-4 & JP-120 X JS-335 & SIHORE \\
\hline 14. & KDS-722 & AMS-99 X EC-241780 & SANGLI (MH) \\
\hline 15. & MAUS-609 & Himso-1563 X MAUS-71 & PARBANI \\
\hline 16. & NRC-107 & Mutant of NRC-37 & INDORE \\
\hline 17. & MACS-1410 & MAUS-144 X MACS-450 & PUNE \\
\hline 18. & JS-20-53 & JS-97-52 X JS-20-02 & JABALPUR \\
\hline 19. & PS-1543 & PS-1029 X JS-335 X PS-1241 & PANTNAGAR \\
\hline 20. & Himso-1685 & H-330 X HARDEE & PALAMPUR \\
\hline 21. & RVS-2002-22 & NRC-37 X JS-39-05 & SIHORE \\
\hline 22. & RKS-111 & RKS-45 X RKS-24 & KOTA \\
\hline 23. & BAUS-27 & PK-472 X L-119 & RANCHI \\
\hline 24. & RSC-10-17 & MAUS-144 X RAUS-5 & RAIPUR \\
\hline
\end{tabular}


Table.2 DNA amplification profile and polymorphism generated in Glycine max L. Merrill by 18 RAPD primers

\begin{tabular}{|c|c|c|c|c|c|c|}
\hline S. No. & Primer & Molecular & Total no. of & Polymorphic bands & \multirow{2}{*}{ PIC* } \\
\cline { 5 - 6 } & Code & $\begin{array}{c}\text { weight range } \\
(\mathbf{b p})\end{array}$ & $\begin{array}{c}\text { bands amplified } \\
\mathbf{( x )}\end{array}$ & Number & $\begin{array}{c}\text { Frequency } \\
(\mathbf{\%})\end{array}$ & \\
\hline 1. & OPA-01 & $300-2300$ & $\mathbf{1 6}$ & $\mathbf{1 6}$ & 100 & 0.257 \\
\hline 2. & OPC-08 & $350-2100$ & 13 & 13 & 100 & 0.322 \\
\hline 3. & OPD-05 & $300-2000$ & 11 & 11 & 100 & 0.295 \\
\hline 4. & OPD-12 & $250-1200$ & 9 & 9 & 100 & 0.183 \\
\hline 5. & OPE-03 & $200-1300$ & 10 & 10 & 100 & 0.359 \\
\hline 6. & OPF-17 & $200-500$ & 3 & 3 & 100 & 0.218 \\
\hline 7. & OPF-19 & $300-700$ & $\mathbf{2}$ & $\mathbf{2}$ & 100 & $\mathbf{0 . 3 9 9}$ \\
\hline 8. & OPJ-04 & $250-2100$ & 14 & 14 & 100 & 0.269 \\
\hline 9. & OPP-01 & $350-1800$ & 10 & 10 & 100 & $\mathbf{0 . 1 2 6}$ \\
\hline 10. & OPP-02 & $200-2100$ & 13 & 13 & 100 & 0.392 \\
\hline 11. & OPP-04 & $100-2500$ & 14 & 14 & 100 & 0.379 \\
\hline 12. & OPP-05 & $300-1800$ & 12 & 12 & 100 & 0.178 \\
\hline 13. & OPP-06 & $200-2200$ & 10 & 10 & 100 & 0.312 \\
\hline 14. & OPP-07 & $300-1200$ & 7 & 7 & 100 & 0.386 \\
\hline 15. & OPP-08 & $300-1300$ & 7 & 7 & 100 & 0.297 \\
\hline 16. & OPP-09 & $300-800$ & 3 & 3 & 100 & 0.343 \\
\hline 17. & OPP-12 & $1000-1600$ & 3 & 3 & 100 & 0.303 \\
\hline 18. & OPP-16 & $200-1800$ & 7 & 7 & 100 & 0.298 \\
\hline & Total & & $\mathbf{1 6 4}$ & $\mathbf{1 6 4}$ & $\mathbf{1 0 0}$ & $\mathbf{0 . 2 9 5}$ \\
\hline
\end{tabular}

*Polymorphic Information Content

Table.3 Genotype specific unique bands as detected by RAPD primers in Glycine max L. Merrill

\begin{tabular}{|c|c|c|c|c|}
\hline S. No. & Primer code & $\begin{array}{c}\text { Total no. of } \\
\text { unique bands }\end{array}$ & Genotype & $\begin{array}{c}\text { Size of band } \\
\text { (bp) }\end{array}$ \\
\hline 1. & OPJ-04 & 1 & JS-20-79 & 2100 \\
\hline $\mathbf{2 .}$ & OPP-05 & 1 & PS-1543 & 800 \\
\hline 3. & OPD-05 & 1 & NRC-98 & 400 \\
\hline 4. & OPP-06 & 1 & Himso-1685 & 200 \\
\hline Total & \multicolumn{4}{|c}{} \\
\hline
\end{tabular}


Table.4 Jaccards similarity coefficient for RAPD profile of Glycine max L. Merrill genotypes

\begin{tabular}{|c|c|c|c|c|c|c|c|c|c|c|c|c|c|c|c|c|c|c|c|c|c|c|c|c|}
\hline Genotypes & 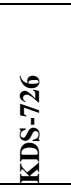 & $\begin{array}{l}\stackrel{\tilde{n}}{n} \\
\dot{\alpha}\end{array}$ & $\begin{array}{l}\stackrel{0}{0} \\
\stackrel{0}{0} \\
\dot{n} \\
0\end{array}$ & ஜ̊ & 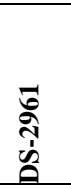 & 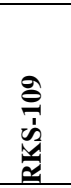 & $\frac{n}{2}$ & $\begin{array}{l}\text { 离 } \\
\text { : } \\
\stackrel{n}{n}\end{array}$ & 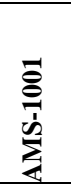 & 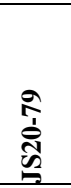 & 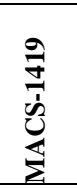 & 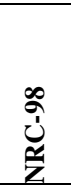 & 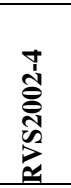 & 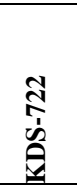 & 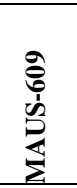 & 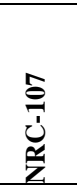 & 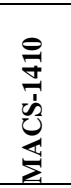 & 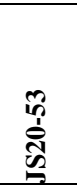 & 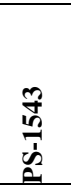 & 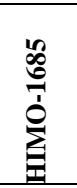 & 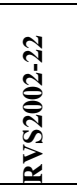 & 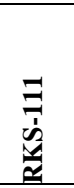 & 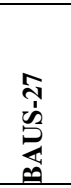 & 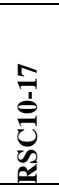 \\
\hline KDS-726 & 1.00 & & & & & & & & & & & & & & & & & & & & & & & \\
\hline PS-1539 & 0.27 & 1.00 & & & & & & & & & & & & & & & & & & & & & & \\
\hline$\overline{D S-3050}$ & 0.41 & 0.35 & 1.00 & & & & & & & & & & & & & & & & & & & & & \\
\hline SL-983 & 0.44 & 0.31 & 0.58 & 1.00 & & & & & & & & & & & & & & & & & & & & \\
\hline DS-2961 & 0.44 & 0.35 & 0.61 & 0.70 & 1.00 & & & & & & & & & & & & & & & & & & & \\
\hline RKS-109 & 0.39 & 0.38 & 0.52 & 0.60 & 0.57 & 1.00 & & & & & & & & & & & & & & & & & & \\
\hline SL-955 & 0.30 & 0.15 & 0.32 & 0.43 & 0.44 & 0.36 & 1.00 & & & & & & & & & & & & & & & & & \\
\hline DS-3047 & 0.42 & 0.18 & 0.49 & 0.49 & 0.47 & 0.46 & 0.39 & 1.00 & & & & & & & & & & & & & & & & \\
\hline AMS-1001 & 0.36 & 0.32 & 0.51 & 0.59 & 0.65 & 0.55 & 0.43 & 0.52 & 1.00 & & & & & & & & & & & & & & & \\
\hline JS20-79 & 0.39 & 0.27 & 0.44 & 0.65 & 0.54 & 0.48 & 0.39 & 0.53 & 0.56 & 1.00 & & & & & & & & & & & & & & \\
\hline MACS-1419 & 0.26 & 0.12 & 0.24 & 0.35 & 0.34 & 0.29 & 0.58 & 0.36 & 0.37 & 0.33 & 1.00 & & & & & & & & & & & & & \\
\hline NRC-98 & 0.26 & 0.14 & 0.23 & 0.36 & 0.33 & 0.30 & 0.49 & 0.27 & 0.32 & 0.27 & 0.44 & 1.00 & & & & & & & & & & & & \\
\hline RVS2002-4 & 0.37 & 0.32 & 0.43 & 0.41 & 0.37 & 0.35 & 0.19 & 0.36 & 0.39 & 0.33 & 0.18 & 0.24 & 1.00 & & & & & & & & & & & \\
\hline KDS-722 & 0.39 & 0.28 & 0.36 & 0.42 & 0.44 & 0.43 & 0.25 & 0.39 & 0.42 & 0.38 & 0.20 & 0.30 & 0.46 & 1.00 & & & & & & & & & & \\
\hline MAUS-609 & 0.44 & 0.35 & 0.45 & 0.49 & 0.53 & 0.48 & 0.29 & 0.40 & 0.49 & 0.39 & 0.24 & 0.31 & 0.49 & 0.68 & 1.00 & & & & & & & & & \\
\hline NRC-107 & 0.27 & 0.17 & 0.29 & 0.33 & 0.31 & 0.33 & 0.50 & 0.32 & 0.34 & 0.27 & 0.56 & 0.34 & 0.20 & 0.23 & 0.33 & 1.00 & & & & & & & & \\
\hline MACS-1410 & 0.20 & 0.14 & 0.26 & 0.37 & 0.32 & 0.33 & 0.31 & 0.29 & 0.34 & 0.27 & 0.25 & 0.29 & 0.22 & 0.20 & 0.29 & 0.27 & 1.00 & & & & & & & \\
\hline JS20-53 & 0.27 & 0.22 & 0.33 & 0.36 & 0.35 & 0.40 & 0.23 & 0.24 & 0.30 & 0.25 & 0.17 & 0.23 & 0.30 & 0.28 & 0.32 & 0.27 & 0.47 & 1.00 & & & & & & \\
\hline PS-1543 & 0.27 & 0.19 & 0.33 & 0.46 & 0.39 & 0.41 & 0.36 & 0.37 & 0.40 & 0.38 & 0.31 & 0.32 & 0.26 & 0.25 & 0.35 & 0.36 & 0.57 & 0.51 & 1.00 & & & & & \\
\hline HIMSO-1685 & 0.23 & 0.17 & 0.33 & 0.40 & 0.37 & 0.36 & 0.35 & 0.29 & 0.35 & 0.30 & 0.27 & 0.29 & 0.27 & 0.27 & 0.31 & 0.34 & 0.53 & 0.60 & 0.69 & 1.00 & & & & \\
\hline RVS2002-22 & 0.22 & 0.18 & 0.29 & 0.38 & 0.35 & 0.36 & 0.32 & 0.34 & 0.38 & 0.32 & 0.29 & 0.27 & 0.22 & 0.22 & 0.31 & 0.30 & 0.62 & 0.51 & 0.65 & 0.65 & 1.00 & & & \\
\hline RKS-111 & 0.21 & 0.17 & 0.29 & 0.36 & 0.34 & 0.37 & 0.30 & 0.32 & 0.34 & 0.28 & 0.23 & 0.28 & 0.26 & 0.24 & 0.30 & 0.33 & 0.56 & 0.58 & 0.65 & 0.61 & 0.70 & 1.00 & & \\
\hline BAUS-27 & 0.29 & 0.23 & 0.24 & 0.30 & 0.31 & 0.25 & 0.20 & 0.20 & 0.27 & 0.26 & 0.14 & 0.18 & 0.27 & 0.25 & 0.33 & 0.19 & 0.34 & 0.42 & 0.38 & 0.37 & 0.34 & 0.36 & 1.00 & \\
\hline RSC10-17 & 0.26 & 0.17 & 0.30 & 0.36 & 0.30 & 0.35 & 0.26 & 0.27 & 0.29 & 0.28 & 0.21 & 0.21 & 0.22 & 0.27 & 0.25 & 0.29 & 0.31 & 0.50 & 0.47 & 0.47 & 0.39 & 0.47 & 0.41 & 1.00 \\
\hline
\end{tabular}


Fig.1 RAPD profile of Glycine max L. Merrill generated through OPP-01 and OPP-04 primer respectively

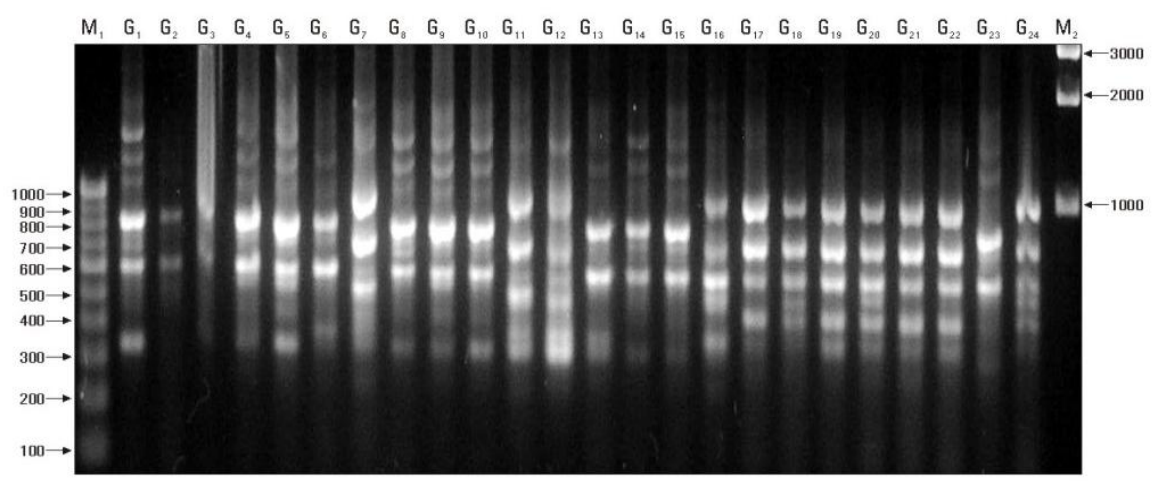

RAPD profile generated through primer - OPP-01

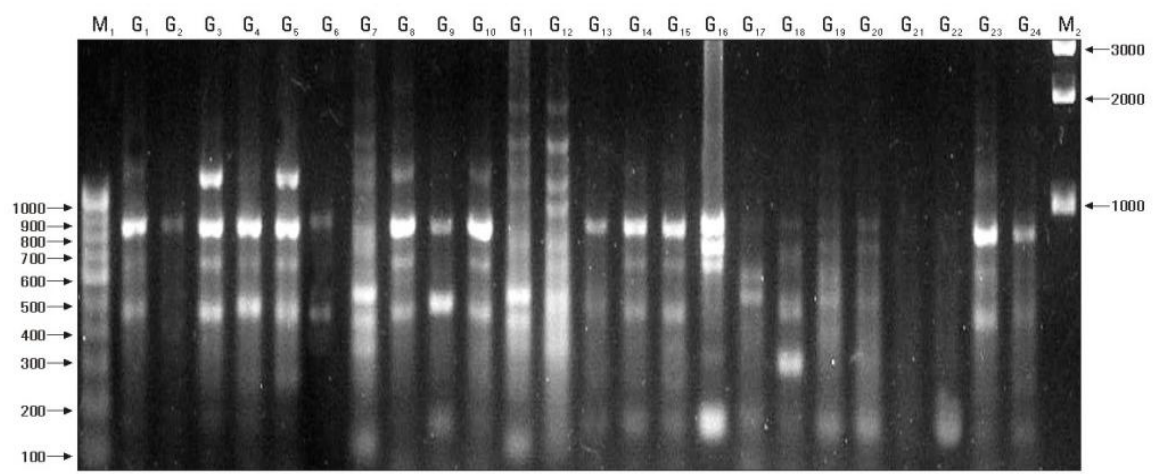

RAPD profile generated through primer - OPP-04

$$
\mathbf{M}_{1}=100 \text { bp DNA Ladder } \quad \mathbf{M}_{2}=1000 \text { bp DNA Ladder }
$$

$\mathrm{G}_{1}-\mathrm{G}_{24}$ represent following Glycine $\max$ genotypes :

\begin{tabular}{|c|c|c|c|c|}
\hline $\mathrm{G}_{1}-\mathrm{KDS}-726$ & $\mathrm{G}_{2}-\mathrm{PS}-1539$ & $\mathrm{G}_{3}-\mathrm{DS}-3050$ & $\mathrm{G}_{4}-\mathrm{SL}-983$ & $\mathrm{G}_{5}-\mathrm{DS} 2961$ \\
\hline $\mathrm{G}_{6}-\mathrm{RKS}-109$ & $\mathrm{G}_{7}-\mathrm{SL}-955$ & $G_{8}-D S-3047$ & $\mathrm{G}_{9}-\mathrm{AMS}-1001$ & $\mathrm{G}_{10}-\mathrm{JS}-20-79$ \\
\hline $\mathrm{G}_{11}-\mathrm{MACS}-1419$ & $\mathrm{G}_{12}-\mathrm{NRC}-98$ & $\mathrm{G}_{13}$ - RVS-2002-4 & $\mathrm{G}_{14}-\mathrm{KDS}-722$ & $\mathrm{G}_{15}$ - MAUS-609 \\
\hline $\mathrm{G}_{16}-\mathrm{NRC}-107$ & $\mathrm{G}_{17}-\mathrm{MACS}-1410$ & $\mathrm{G}_{18}-\mathrm{JS}-20-53$ & $\mathrm{G}_{19}-\mathrm{PS}-1543$ & $\mathrm{G}_{20}$ - Himso- 1685 \\
\hline$G_{21}-$ RVS-2002-22 & $\mathrm{G}_{22}-\mathrm{RKS}-111$ & $\mathrm{G}_{23}-$ BAUS- 27 & $\mathrm{G}_{24}-\mathrm{RSC}-10-17$ & \\
\hline
\end{tabular}


Fig.2 Dendogram constructed with UPGMA clustering method of 24 Glycine max

L. Merrill genotypes using RAPD primers

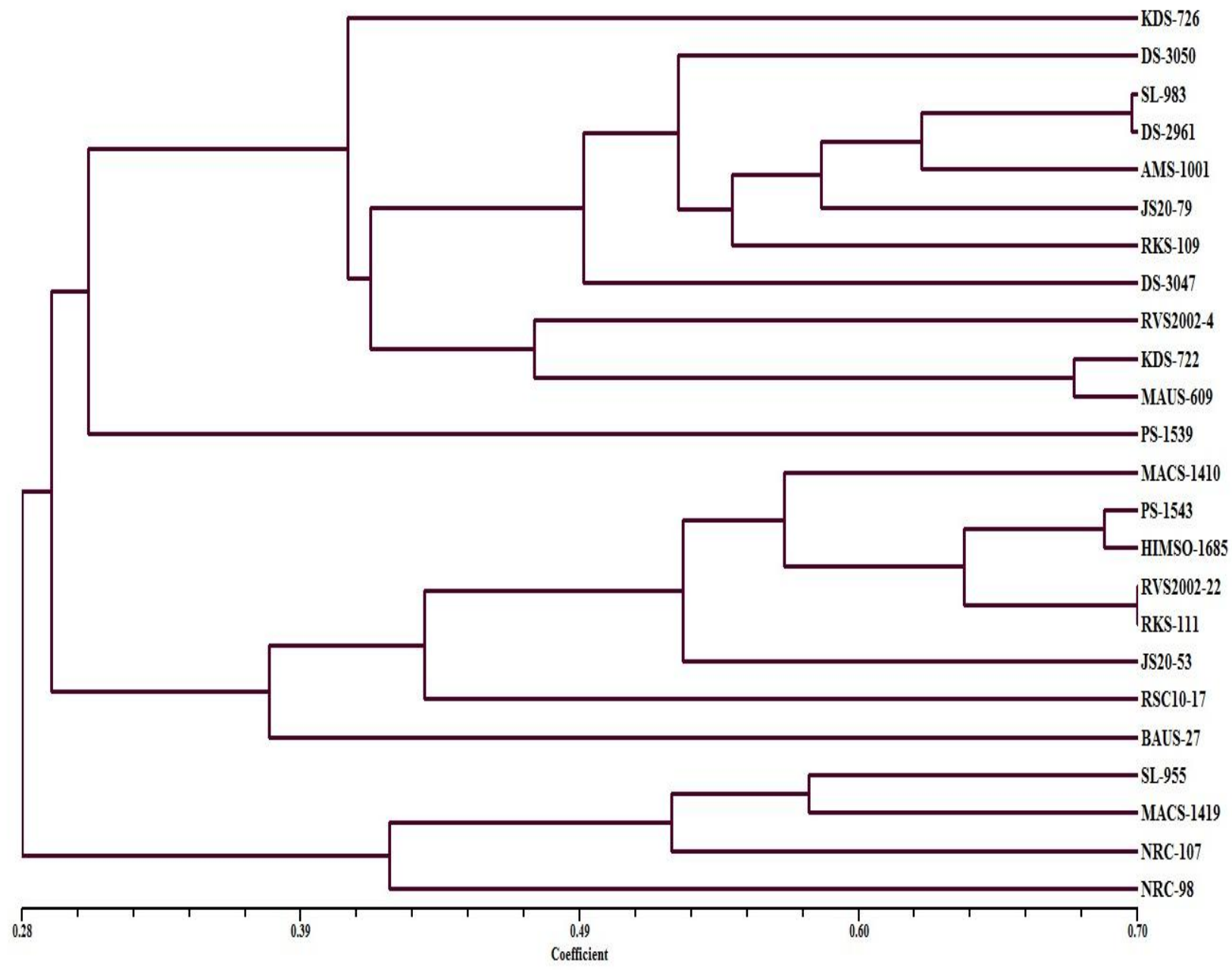

Fig.3 Two dimensional PCA (Principle Component Analysis) scaling of 24 genotypes of Glycine $\max$ L. Merrill using RAPD markers

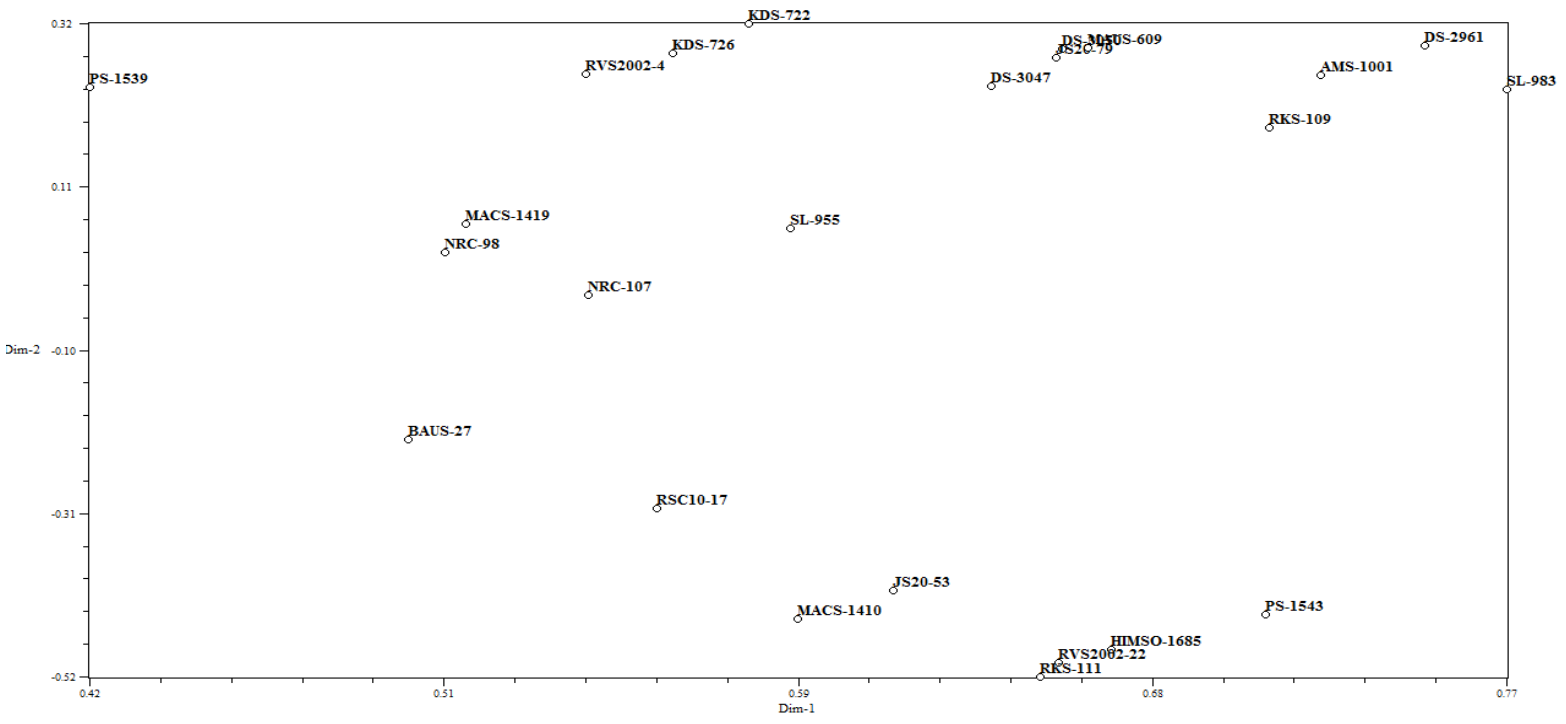


Fig.4 Three dimensional PCA (Principle Component Analysis) scaling of 24 genotypes of Glycine max L. Merrill using RAPD markers

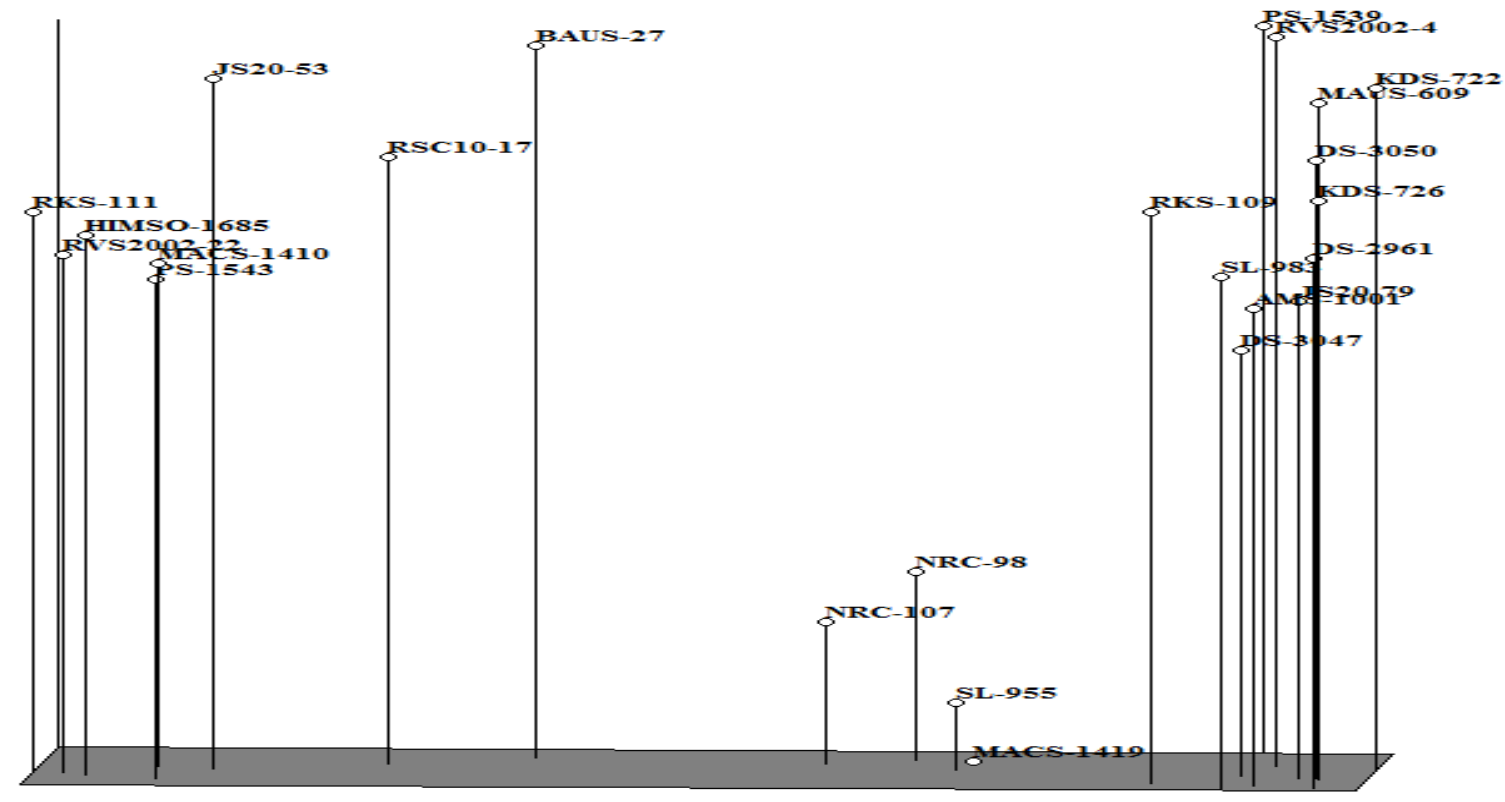

First sub-cluster consisted of two genotypes viz., PS-1543 and Himso-1685 which were similar to each other at a similar coefficient of 0.69 . The second sub-cluster contained two genotypes named RVS-2002-22 and RKS-111. These were related to each other at a similarity coefficient of 0.70 . The cluster III included 4 genotypes viz., SL955, MACS-1419, NRC-107 and NRC-98 at a similarity coefficient of 0.42 . It could be divided into one sub-cluster. This subcluster included genotypes SL-955 and MACS-1419 which were similar to each other at similarity coefficient of 0.58 .

Two and three dimension principal component analysis based on RAPD data (Figs. 3 and 4, respectively) showed similar clustering of 24 genotypes as evident from cluster tree analysis. Dice similarity coefficients ranged from 0.42 to 0.77 . Most of the genotypes tended to cluster mainly into three clusters. Cluster I included 12 genotypes (KDS-726, DS-3050, SL-983, DS-2961, AMS-1001, JS-20-79, RKS-109,
DS3047, RVS2002-4, KDS-722, MAUS609 and PS-1539) second included 8 genotypes MACS-1410, PS-1543, HIMSO1685, RVS2002-22, RKS-111, JS20-53, RSC10-17 and BAUS-27) and Cluster III included 4 genotypes viz., (SL-955, MACS1419, NRC-107 and NRC-98).

In present study, we found that all the primers studied produced $100 \%$ polymorphism, relatively high proportion compared to previous reports such as Khare et al., (2013) (97.68\%), Mundewadikar and Deshmukh (2014) (94.06\%) and Singh et al., (2008) (89.9\%).

The RAPD methods displayed genetic variation among 24 soybean genotypes and phylogenetic tree was showing a relationship among them. This study has confirmed, RAPD marker is potentially simple, rapid, reliable and effective method of detecting polymorphism for assessing genetic diversity among genotypes. The banding pattern obtained from RAPD 
markers can be used to characterize soybean genotypes. It is observed that there is a wide range of genetic diversity among selected genotypes, thus they can be used for further crop improvement programmes.

\section{Acknowledgement}

Authors are very gratefully acknowledged the financial assistance from RKVY project "Validation of important crop varieties through DNA fingerprinting".

\section{References}

Antalina. 1999. Recent Research and Industrial achievement for soybean in Japan. Proceeding of RIELT-JIRCAS. Workshop on Soy Res., Sep. 28.

Bharose, A.A., Kulkarni, V.D. and Damse, D.N. 2017. Molecular Diversity Analysis of Soybean Genotypes Using Molecular Markers. Int. J. Curr. Microbiol. App. Sci., 6(3): 1723-1729.

Brown-Guedira, G.L., Thompson, J.A., Nelson, R.L. and Warburton, M.L. 2000. Evaluation of genetic diversity of soybean introductions and North American ancestors using RAPD and SSR markers. Crop Sci., 40: 815-823.

Chen, L.F.O., Yun, W.C., Kou, H.Y. and Chen, M.H. 1994. Polymorphic distinction of soybean by molecular markers. Soybean Genet. Newsl., 21: 7075.

Chowdhury, A.K., Srinives, P., Tongpamnak P. and Saksoon, P. 2001. Genetic diversity based on morphology and RAPD analysis in vegetable soybean. Korean J. Crop Sci., 46(2): 112-120.

Doyle, J.J. and Doyle, J.L. 1990. Isolation of DNA from fresh plant tissue. Focus, 12: 13-15.

Dwivedi, S.L., Gurtu, S., Chandra, S., Yuejin, W. and Nigam, S.N. 2001. Assessment of genetic diversity among selected groundnut germplasm by RAPD analysis. Plant Breeding, 120: 345-350.
Fahima, T., Sun, G.L., Beharav, A., Krugman, T., Beiles, A. and Nevo, E. 1999. RAPD polymorphism of wild emmer wheat populations, Triticum dicoccoides, in Israel. Theor. Appl. Genet., 98: 434-447.

Fukuda, Y. 1933. Cytogenetical studies on the wild and cultivated Manchurian soybean. Jpn. J. Bot., 6.

Garcia, G.M., Stalker, H.T., Schroeder, E., Lyerly, J.H. and Kochert, G. 2005. A RAPD-based linkage map of peanut based on a backcross population between the two diploid species Arachissteno sperma and A. cardenasii. Peanut Sci., 32: 1-8.

$\mathrm{Hu}$, J. and Quiros, C.F. 1991. Identification of broccoli and cauliflower cultivars with RAPD markers. Plant Cell Rep., 10: 505- 511.

Jain, A., Bhatia, S., Banga, S.S., Prakash, S. and Lakshmikumaram, M. 1994. Theoret. Appl. Gen., 88: 116- 122.

Khare, D., Bisen, A., Nair, P. and Tripathi, P. 2013. Genetic diversity in soybean germplasm identified by RAPD markers. Asia-Pac. J. Mol. Biol., 21(3): 121-123.

Kumari, V., Gowda, M.V.C. and Bhat, R. 2009. Molecular characterization of induced mutants in groundnut using Random Amplified Polymorphic DNA markers. Karnataka J. Agricult. Sci., 22: 276-279.

Li, Z. and Nelson, R.L. 2001. Genetic diversity among soybean accessions from three countries measured by RAPD. Crop Sci., 41: 1337-1347.

Li, Z., Qui, L., Thompson, J.A., Welsh, M. M. and Nelson, R.L. 2001. Molecular genetic analysis of U.S. and Chinese soybean ancestral lines. Crop Sci., 37: 605-613.

Mignouna, H.D., Ng, N.Q., Ikca, J. and Thottapilly, G. 1998. Genetic diversity in cowpea as revealed by random amplified polymorphic DNA. J. Genet. Breed, 52: 151-159.

Mundewadikar, D.M. and Deshmukh, P.R. 2014. Genetic Variability and Diversity 
Studies in Soybean [Glycine max (L.) Merrill] using RAPD Marker. IJSRP, 9(4): ISSN 2250-3153.

Ojo, D.K., Ajayi, A.O. and Oduwaye, O.A. 2012. Genetic Relationships among Soybean Accessions Based on Morphological and RAPDs Techniques. Pertanika J. Trop. Agric. Sci., 35: 237 24.

Paterson, A.H., Damon, S., Hewitt, J.D., Zamir, S., Rabinowitch, H.D., Lincoin, S.E., Lander, S.E. and Tanksley, S.D. 1991. Mendelian factors underlying quantitative traits in tomato: comparison across species, generations, and environments. Genetics, 127: 181-197.

Singh, R.K., Kumar, A., Billore, M., Rani, A., Husain, S.M. and Chauhan, G. S. 2006. Analysis of Soybean Germplasm using Randomly Amplified Polymorphic DNA Markers. The Nucleus, 49: 165172.

Singh, R.K., Satyawathi, C.T., Rani, A. and Chandra, A. 2010. Genetic diversity in Indian ancestors, cultivars and exotic germplasm of soybean as revealed by RAPD markers. Natl. Acad. Sci. Lett., 33(1/2): 1-8.

Subudhi, P.K and Huang, N. 1999. RAPD mapping in a doubled haploid population of rice (Oryza sativa L.). Hereditas, 130: 2-9.

Tahir, N.A.R. and Karim, H.F.H. 2011. The Determination of Genetic Relationship among some varieties of Chickpea $(C$. arietinum L.) in Sulaimani by RAPD and ISSR markers. Jordan J. Biol. Sci., 4(2): 77-86.

Thompson, J.A. and Nelson, R.L. 1998. Utilization of diverse germplasm for soybean yield improvement. Crop Sci., 38: 1362-1368.

Thompson, J.A., Nelson, R.L. and Vodkin, L.D. 1998. Identification of diverse soybean germplasm using RAPD markers. Crop Sci., 38: 1348-1355.

Williams, G.K., Kubelik, A.R., Livak, K.L., Rafalshi, J.A. and Tingey, S.V. 1990. DNA polymorphisms amplified by arbitrary primers are useful as genetic markers. Nucleic Acids Res., 18: 65316535.

Winter, P. and Kahl, G. 1995. Molecular marker technologies for plant improvement. World J. Microb. Biot., 11: 438-448.

Yu, C.Y., Hu, S.W., Zhao, H.X., Guo, A. G. and Sun, G.L. 2005. Genetic distances revealed by morphological characters, isozymes, proteins and RAPD markers and their relationships with hybrid performance in oilseed rape (Brassica napus L.). Theor. Appl. Genet., 110(3): 511-518.

Zenglu, L. and Nelson, R.L. 2002. RAPD Marker Diversity among Cultivated and Wild Soybean Accessions from Four Chinese Provinces. Crop Sci., 42: 17371744.

\section{How to cite this article:}

Ravindra Kumar Jain, Arunabh Joshi and Devendra Jain. 2017. Molecular Marker Based Genetic Diversity Analysis in Soybean [Glycine max (L.) Merrill] Genotypes. Int.J.Curr.Microbiol.App.Sci. 6(6): 1034-1044. doi: https://doi.org/10.20546/ijcmas.2017.606.119 